\title{
Factsheet: Youth in India: Situation and needs study-Key indicators, 2006-2007: Bihar
}

International Institute for Population Sciences (IIPS)

Follow this and additional works at: https://knowledgecommons.popcouncil.org/departments_sbsr-pgy

Part of the Demography, Population, and Ecology Commons, Family, Life Course, and Society Commons, International Public Health Commons, and the Medicine and Health Commons How does access to this work benefit you? Let us know!

\section{Recommended Citation}

"Factsheet: Youth in India: Situation and needs study-Key indicators, 2006-2007: Bihar." New Delhi: Population Council, 2008. 


\section{Youth in India: Situation and Needs Study}

\section{Key Indicators, 2006-2007: Bihar}

\section{Socio demographic profile}

\begin{tabular}{ccccccccccccc}
\multicolumn{4}{c}{ Combined } & \multicolumn{4}{c}{ Urban } \\
\hline MM & MF & UM & UF & MM & MF & UM & UF & MM & MF & UM & UF
\end{tabular}

1. Co-reside with a parent (\%)

2. Have completed 8 years of schooling (\%)

3. Were not in school at age $12(\%)$

4. Engaged in paid and/or unpaid work in last 12 months (\%)

5. Engaged in paid work in last $\mathbf{1 2}$ months (\%)

6. Unemployment rate (as percentage of labour force)

7. Unemployment rate among youth with 12 years of schooling'

8. Were both working and in school at age $15(\%)$

9. Report that one parent or both parents discussed sexual and reproductive systems of men and women and/or how

ondent (\%)

10. Report talking to one parent or both parents about friends (\%)

Young people's control over their own lives

11. Have a bank account (\%)

12. Take independent decisions about buying clothes (\%)

13. Allowed to visit friends inside village/area unescorted (\%)

14. Allowed to visit health facility unescorted $(\%)$

Sexual and reproductive health knowledge

15. Correctly reported legal age at marriage for girls (\%)

16. Know that a woman can get pregnant

at very first sexual Intercourse (\%)

17. Have heard about:

a. Condom (\%)

b. Oral contraceptive pills $(\%)$

c. IUD (\%)

d. Withdrawal (\%)

18. Have correct specific knowledge ${ }^{2}$ of:

a. Condom (\%)

b. Oral contraceptive pills $(\%)$

c. IUD (\%)

d. Withdrawal (\%)

19. Report that condom does not reduce sexual pleasure (\%)

20. Have comprehensive knowledge about legality of abortion ${ }^{3}(\%)$

21. Have heard about: a. HIV/AIDS (\%)
b. STI/RTI (\%)

22. Have comprehensive knowledge about $\operatorname{HIV}^{4}(\%)$

Pre-marital romantic and sexual relationships

23. Ever had an opposite sex romantic partner (\%)
24. Had a first date with opposite sex partner before age $15(\%)$

24. Had a first date with opposite sex partner before age 15
25. Ever had pre-marital sexual relation with opposite sex

25. Ever had pre-marital sex
romantic partner (\%)

26. Ever had pre-marital sex $(\%)^{5}$

27. Report consistent condom use with pre-marital partners ${ }^{6}(\%)$

$\begin{array}{rrrr}66.5 & 14.8 & 94.5 & 96.6 \\ 41.8 & 15.4 & 59.7 & 38.4 \\ 39.0 & 70.3 & 18.0 & 35.0 \\ 97.0 & 38.0 & 64.3 & 36.0 \\ 77.5 & 23.6 & 39.1 & 16.8 \\ 9.8 & 34.5 & 28.8 & 37.6 \\ 26.7 & 86.7 & 71.7 & 90.4 \\ 12.5 & 3.0 & 21.4 & 8.3 \\ & & & \\ 0.6 & 3.4 & 0.1 & 1.5 \\ 57.7 & 59.2 & 62.2 & 72.8\end{array}$

\section{6}

$28.3 \quad 44.1 \quad 13.9 \quad 16.0$

$\begin{array}{llll}94.6 & 12.6 & 53.7 & 17.0\end{array}$

$82.6 \quad 7.7 \quad 41.8$

$\begin{array}{llll}7.1 & 68.2 & 24.6 & 51.2\end{array}$

$\begin{array}{llll}28.6 & 94.1 & 68.8 & 91.2\end{array}$

$12.0 \quad 1.4 \quad 11.4$

3.4

$\begin{array}{llll}1.1 & 4.2 & 0.4 & 3.2\end{array}$

$\begin{array}{llll}55.9 & 69.2 & 66.4 & 82.4\end{array}$

$\begin{array}{rccr}66.3 & 14.9 & 96.1 & 97.0 \\ 40.2 & 13.8 & 56.8 & 33.0 \\ 40.0 & 72.0 & 18.8 & 38.5 \\ 97.2 & 39.7 & 66.4 & 39.5 \\ 77.0 & 24.6 & 38.5 & 18.3 \\ 10.0 & 33.2 & 29.6 & 35.3 \\ 26.6 & * & (73.2) & 90.3 \\ 12.5 & 3.1 & 23.3 & 9.2 \\ & & & \\ 0.6 & 3.4 & 0.0 & 1.2 \\ 57.8 & 58.5 & 61.4 & 71.0\end{array}$

$\begin{array}{llll}32.6 & 12.0 & 20.0 & 15.6\end{array}$

$\begin{array}{llll}83.7 & 40.1 & 61.1 & 50.3\end{array}$

na. $\quad 52.8 \quad 95.9 \quad 74.1$ $\begin{array}{rrrr}16.0 & 3.8 & 8.4 & 3.4 \\ 80.8 & 28.2 & 41.3 & 26.1\end{array}$

na. $\quad 52.6 \quad 94.2 \quad 62.6$

na. $\quad 11.0 \quad 69.6 \quad 4.6$

Self-reported health problems

28. Report suffering from high fever, skin problems or asthma during the past three months (\%)

29. Report any reproductive health problem (\%)

$\begin{array}{rrrr}68.3 & 52.5 & 73.5 & 67.5 \\ 40.0 & 37.0 & 24.2 & 25.6 \\ & & & \\ 92.5 & 67.2 & 88.5 & 50.5 \\ 86.5 & 86.8 & 75.4 & 78.1 \\ 38.4 & 48.9 & 32.3 & 36.3 \\ 6.4 & 42.2 & 2.9 & 2.4 \\ & & & \\ 73.5 & 36.4 & 58.7 & 16.7 \\ 41.2 & 53.4 & 23.1 & 37.3 \\ 17.0 & 27.3 & 8.4 & 12.9 \\ 5.3 & 33.8 & 1.7 & 1.3 \\ 28.6 & 22.7 & 15.9 & 12.7 \\ 6.5 & 2.6 & 5.9 & 3.0 \\ & & & \\ 86.5 & 42.6 & 87.7 & 52.8 \\ 18.9 & 15.8 & 10.6 & 3.7 \\ 27.0 & 13.2 & 27.6 & 18.0\end{array}$

$\begin{array}{rrrr}77.2 & 73.2 & 77.9 & 82.8 \\ 46.7 & 45.8 & 30.7 & 35.4 \\ & & & \\ 97.8 & 85.9 & 96.7 & 80.2 \\ 94.6 & 94.4 & 87.3 & 91.9 \\ 55.4 & 74.1 & 45.7 & 55.5 \\ 10.9 & 51.4 & 4.1 & 3.4 \\ & & & \\ 86.0 & 58.7 & 68.0 & 30.0 \\ 46.7 & 67.6 & 29.5 & 50.7 \\ 30.4 & 47.2 & 13.9 & 24.9 \\ 8.7 & 44.4 & 2.9 & 2.0 \\ 35.2 & 33.6 & 21.1 & 14.9 \\ 6.5 & 3.5 & 7.3 & 3.8 \\ & & & \\ 95.7 & 71.1 & 95.9 & 85.3 \\ 23.9 & 14.1 & 12.7 & 6.9 \\ 40.2 & 32.4 & 42.0 & 40.1\end{array}$

$\begin{array}{rrrr}67.4 & 51.1 & 72.7 & 64.7 \\ 39.4 & 36.4 & 23.0 & 23.8 \\ & & & \\ 92.0 & 66.0 & 86.9 & 45.1 \\ 85.7 & 86.3 & 73.0 & 75.5 \\ 36.9 & 47.2 & 29.7 & 32.8 \\ 6.1 & 41.7 & 2.7 & 2.3 \\ & & & \\ 72.3 & 34.9 & 56.9 & 14.3 \\ 40.7 & 52.5 & 21.8 & 34.9 \\ 15.7 & 26.0 & 7.4 & 10.7 \\ 5.0 & 33.2 & 1.4 & 1.3 \\ 28.0 & 21.8 & 14.8 & 12.0 \\ 6.5 & 2.5 & 5.6 & 2.8 \\ & & & \\ 85.7 & 40.8 & 86.0 & 46.8 \\ 18.5 & 15.9 & 10.1 & 3.1 \\ 25.8 & 12.0 & 24.8 & 13.9\end{array}$

\section{Youth life style}

30. Consumed alcohol at least once in the last month (\%)

31. Consumed drugs at least once in the last month (\%)

32. Consumed tobacco products at least once in the last month (\%)

33. Involved in physical fights in last 12 months (\%)

34. Often watch television (\%)

$\begin{array}{rrrrrrrrrrrr}16.8 & 3.6 & 16.0 & 7.1 & 19.6 & 9.2 & 20.5 & 9.5 & 16.5 & 3.3 & 15.1 & 6.7 \\ 4.4 & 2.0 & 4.5 & 2.2 & 3.3 & 2.8 & 4.9 & 2.2 & 4.4 & 1.9 & 4.4 & 2.2 \\ & & & & & & & & & & \\ 8.8 & 1.3 & 6.2 & 1.8 & 5.4 & 2.1 & 3.7 & 0.8 & 9.1 & 1.3 & 6.7 & 2.0 \\ 18.0 & 2.4 & 10.7 & 3.0 & 16.3 & 2.8 & 8.2 & 1.6 & 18.2 & 2.4 & 11.3 & 3.3 \\ 4.5 & 0.0 & 9.6 & 4.5 & 0.0 & (0.0) & (14.3) & * & 4.8 & * & 9.1 & (4.8)\end{array}$

\section{Programme participation and voting experience}

community in the last three years (\%)

36. Youth aged 18 years or more who voted in the last election (\%)

6.8
77.

$0.0 \quad(0.0) \quad(14.3)$

4.8

Married life

37. Married before legal age at marriage (\%)

38. Report a love marriage (\%)

39. Co-reside with a parent-in-law (\%)

40. Usually discuss money matters with spouse $(\%)$

41. Report any physical violence perpetrated on wife by husband (\%)

42. Ever had forced sex with spouse ${ }^{7}(\%)$

43. Ever had extra-marital sex (\%)

44. Ever used contraception within marriage (\%)

45. Currently using contraception (\%)

46. Ever used a method to delay first pregnancy (\%)

47. Children ever born (mean)

48. Ideal number of children ${ }^{8}$ (mean)

49. First birth attended by health professional ${ }^{9}(\%)$

$\begin{array}{rrrr}28.0 & 33.2 & 31.0 & 27.8 \\ 10.6 & 31.7 & 7.0 & 19.4\end{array}$

$\begin{array}{llll}26.9 & 34.5 & 31.8 & 31.8\end{array}$

18.4

18.4

$\begin{array}{llll}28.1 & 33.2 & 30.8 & 27.1\end{array}$

$\begin{array}{rrrrrrrrrrrr}26.0 & 0.1 & 6.7 & 0.1 & 31.5 & 0.0 & 7.8 & 0.0 & 25.6 & 0.1 & 6.5 & 0.1 \\ 2.8 & 0.0 & 0.7 & 0.0 & 2.2 & 0.0 & 0.8 & 0.0 & 2.9 & 0.0 & 0.7 & 0.0 \\ 67.4 & 1.7 & 28.7 & 1.2 & 69.6 & 1.4 & 27.9 & 0.4 & 67.2 & 1.7 & 28.8 & 1.3 \\ 10.5 & 4.4 & 15.3 & 3.7 & 12.0 & 2.8 & 15.2 & 2.0 & 10.3 & 4.5 & 15.3 & 4.0 \\ 5.7 & 5.6 & 11.6 & 13.4 & 25.3 & 34.5 & 34.3 & 48.6 & 3.9 & 3.7 & 7.2 & 6.9\end{array}$


